\title{
Liver Abscess Metastatic Syndrome Caused by Hypermucoviscous Klebsiella Pneumoniae in a Canadian Patient of Vietnamese Origin
}

Sandra Criales, MD, Alexandre Lafleur, MD, MSc (Ed), Phillipe Gervais, MD

\begin{abstract}
About the Authors
Dr. Sandra Criales is a 4th-year infectiology-microbiology resident at Université Laval (Quebec City, Canada). Dr. Alexandre Lafleur is assistant clinical professor of medicine at Université Laval and specialist in general internal medicine at CHU de Québec. Dr. Phillipe Gervais is an infectious diseases specialist at CHU de Québec and Quebec Heart and Lung Institute. This article was written in collaboration with the QMA-CMA-MD Educational Leadership Chair in Health Professions Education at Université Laval Faculty of Medicine.
\end{abstract}

Correspondence can be directed to: Sandra-Patricia.Criales-Doria.1@ulaval.ca

Submitted: March 3, 2018. Accepted: May 11, 2018. Published: February 12, 2019. DOI: 10.22374/cjgim.v14i1.278

\begin{abstract}
Klebsiella pneumoniae liver abscess syndrome (KLAS) is an emerging infection caused by hypermucoviscous strains (K1, rmpA, mgA) with a particular virulence at risk of metastatic dissemination. We describe a case of metastatic KLAS in a Canadian immunocompetent patient of Vietnamese origin who presented with fever and abnormal liver function tests. Imaging studies revealed unique liver and pulmonary abscesses. Blood and liver abscess cultures showed colonies of K.pneumoniae with hypermucoviscous phenotype, a K1 serotype and the presence of a rmpA gene confirming biomolecular features of the invasive syndrome. Mostly reported in patients of Asian origin, KLAS has been reported in Canada since 2007. Prompt identification and treatment prevents severe complications such as endophthalmitis, meningitis, lung abscess and spondylodiscitis.
\end{abstract}

\section{Résumé}

Le syndrome d'abcès hépatique à Klebsiella pneumoniae (KLAS en anglais) est une infection en émergence résultant d'une souche hypermuqueuse (K1, rmpA, mgA) d'une virulence accrue, à risque de dissémination. Nous décrivons un cas de KLAS métastatique chez un patient canadien d’origine vietnamienne, immunocompétent, qui présentait de la fièvre et des anomalies du bilan hépatique. Les imageries ont révélé des abcès hépatiques et pulmonaires uniques. Les hémocultures et les cultures du drainage de l'abcès hépatique ont confirmé la présence d'une souche hypermuqueuse de Klebsiella pneumoniae, sérotype K1, génotype rmpA, caractéristiques biomoléculaires associées aux infections invasives. Principalement décrits chez des patients d’origine asiatique, des cas de KLAS sont rapportés au Canada depuis 2007. L'identification et le traitement rapide préviendront des complications sévères, dont lendophtalmite, la méningite, l'abcès pulmonaire et la spondylodiscite.

Keyword: liver abscess metastatic syndrome, hypermucoviscous klebsiella pneumoniae 
In March 2015, a 34-year-old patient presented at the emergency department of CHU de Québec (CHUL) and was admitted to the internal medicine clinical teaching unit. He reported one week of fever and chills, profuse sweating, temporal headache, loss of appetite, and myalgia. The patient emigrated from Vietnam to Canada 11 years earlier and had not returned there since then. His only recent trip had been to Boston in 2011. He completed a treatment for latent tuberculosis in 2005. He worked as an informatician, had no allergies, and did not take medication. The patient reported no infectious contact, illicit drug abuse, or other at-risk exposures for malaria, viral hepatitis, Q fever, leptospirosis, or brucellosis. The patient did not complain of rash, weight loss, or any abdominal, pulmonary, urinary, or neurological symptoms.

The patient was diaphoretic without rash or jaundice. Physical findings included a blood pressure of 90/58 $\mathrm{mmHg}$, a temperature of $38.9^{\circ} \mathrm{C}$, a heart rate of 100 beats per minute, non-painful cervical lymphadenopathy (less than $2 \mathrm{~cm}$ ), and hepatomegaly ( $2 \mathrm{~cm}$ below costal margin). The remainder of the physical examination was normal without meningeal sings, abdominal mass, tenderness, or ascites. Vital signs stabilized rapidly with treatment and intravenous fluids.

Laboratory studies showed hyponatremia at $131 \mathrm{mmol} / \mathrm{L}$ (135-145 mmol/L), white blood cell count at $9.9 \times 10^{9}$, thrombocytopenia at $50 \times 10^{9} \mathrm{~g} / \mathrm{L}\left(150-400 \times 10^{9} \mathrm{~g} / \mathrm{L}\right)$, abnormal liver and pancreas functions tests: aspartate aminotransferase $0.68 \mu \mathrm{kat} / \mathrm{L}(<0.67 \mu \mathrm{kat} / \mathrm{L})$, gamma-glutamyl transferase 3.57 $\mu \mathrm{kat} / \mathrm{L}(<0.83 \mu \mathrm{kat} / \mathrm{L})$, total bilirubin $31 \mu \mathrm{mol} / \mathrm{L}(0-21 \mu \mathrm{mol} / \mathrm{L})$, direct bilirubin $16 \mu \mathrm{mol} / \mathrm{L}(<4 \mu \mathrm{mol} / \mathrm{L})$ amylase $2.99 \mu \mathrm{kat} / \mathrm{L}$ (<1.67 $\mu \mathrm{kat} / \mathrm{L})$, lipase $4.21 \mu \mathrm{kat} / \mathrm{L}(<0.92 \mu \mathrm{kat} / \mathrm{L})$. Tests for amebiasis, echinococcosis, human immunodeficiency virus, and hepatitis B-C were negative and tests for Epstein-Barr virus and cytomegalovirus showed an ancient exposition.

Abdominal ultrasound, contrast-enhanced computerized tomography (CT) scan, and magnetic resonance imaging (MRI) showed a unique hepatic abscess with a diameter of $3.3 \mathrm{~cm}$ in the eighth segment of the liver (Figure 1). A chest CT revealed an asymptomatic pulmonary abscess in the right lower lobe (Figure 2).

Blood cultures and cultures from percutaneous drainage of the hepatic abscess grew Klebsiella pneumoniae with hypermucoviscous phenotype, defined by a positive 'string test' (viscous string > $5 \mathrm{~mm}$ when bacterial colonies on an agar plate are stretched by an inoculation loop) as shown in Figure 3. The Canadian Science Centre for Human and Animal Health (Winnipeg, Canada) confirmed a K1 serotype and the presence of a rmpA gene confirming biomolecular features of the invasive syndrome.

The pulmonary metastatic lesion was the hallmark for the diagnostic of Klebsiellapneumoniae liver abscess metastatic

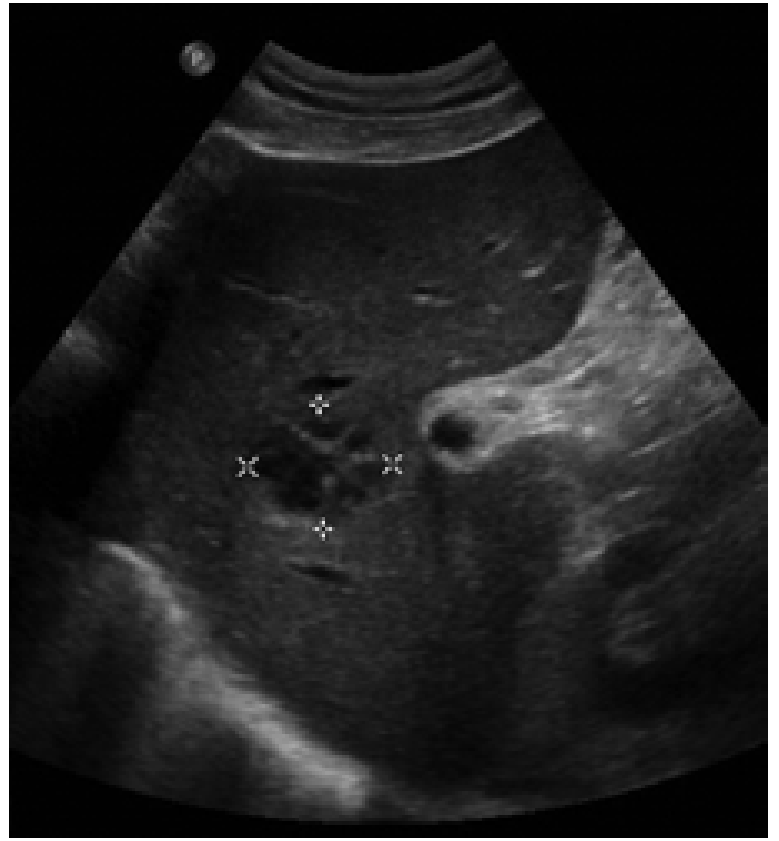

Figure 1. Abdominal ultrasound showing a $3.3-\mathrm{cm}$ abscess in the eighth segment of the liver.

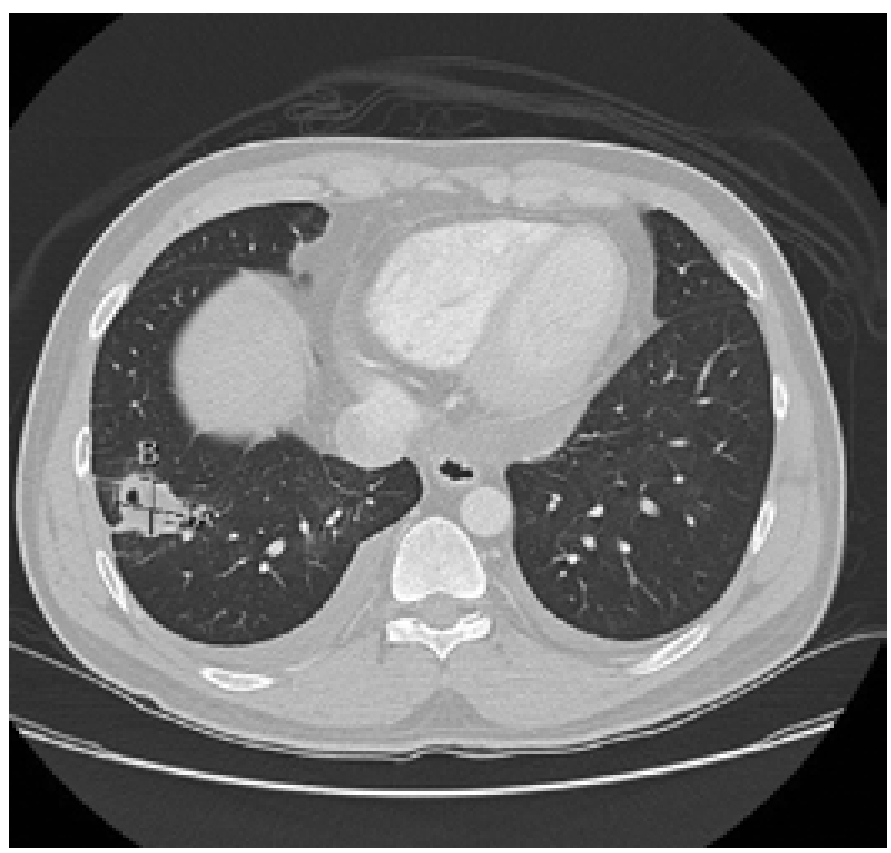

Figure 2. Chest computed tomography scan revealing a $2.9 \times 2.3 \mathrm{~cm}$ pulmonary abscess in the right lower lobe.

syndrome (KLAS). Based on in vitro susceptibilities, piperacillintazobactam, empirically given, was replaced by ceftriaxone for six weeks of IV ambulatory treatment. The liver abscess, of less than $5 \mathrm{~cm}$, was entirely drained with a single percutaneous needle aspiration, without surgery. Drainage was not indicated for the uncomplicated pulmonary abscess. A cerebral MRI did not show endophthalmitis, abscess or meningeal anomalies. 


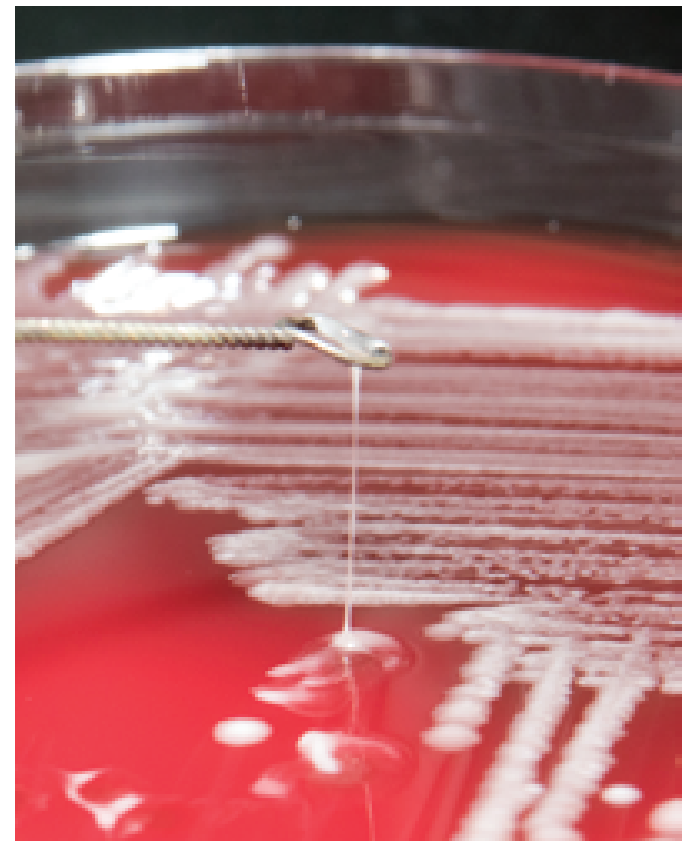

Figure 3. Patient's isolated colonies plate culture demonstrating a positive 'string test' (formation of a viscous string $>5 \mathrm{~mm}$ ).

Lumbar puncture was not initially performed because of the thrombocytopenia.

Followed until July 2015 by CT scans, hepatic and pulmonary lesions disappeared, and liver tests normalized after antibiotic treatment. The patient has not relapsed.

\section{Discussion}

Klebsiella pneumoniae is a well-known gram-negative bacillus with a thick polysaccharide. Standard clinical diseases are related to K. pneumoniae and K. oxytoca ${ }^{1-8}$ such as pneumonia, urinary tract infections, abdominal cavity surgery-related infections and several nosocomial syndromes often associated with a history of alcohol abuse or diabetes mellitus.

KLAS is a distinctive infection related to specific subcapsular serotypes and a marked virulence that produces liver abscesses and several distant metastatic infections. ${ }^{1}$ Described mostly in the Asian population since the 1980 s, cases have been diagnosed in North and South America, ${ }^{2-3,8}$ Europe,${ }^{9}$ Canada, ${ }^{10}$ and Australia. ${ }^{11}$

A liver abscess caused by this particular microbiological strain with extra-hepatic infections should be recognized as KLAS. ${ }^{1,4}$ The clinical signs of KLAS are fever, chills, headache, and abdominal pain. Nausea and vomiting occur in about $25 \%$ of patients. ${ }^{1}$ Half of the patients have jaundice and hepatomegaly. ${ }^{8}$ Blood test findings are leucocytosis, increased C-reactive protein, abnormal liver function tests, and thrombocytopenia. ${ }^{1,8}$

Metastatic spread, unusual for most enteric gram-negative bacilli, is a hallmark of the hypermucoviscous Klebsiella pneumonia strain infecting immunocompetent hosts. From 8 to $30 \%$ of metastatic disease in KLAS, ${ }^{1,4,17}$ a case series of 23 patients with metastatic lesions reported mostly endophthalmitis, uveitis, pulmonary abscess, and purulent meningitis. ${ }^{12}$

Hypervirulent Klebsiella pneumoniae (hvKP) is directly linked to the hypermucoviscous phenotype, related to the $\mathrm{k} 1, \mathrm{k} 2$ serotypes and the regulator of mucoid phenotype A (rmpA) gene. ${ }^{4}$ This feature gives this type of pathogen the ability to produce lethal extra liver infections in nonimmunocompromized patients. ${ }^{5-7,18}$ Numerous virulence factors have been elucidated and implicate the presence of mucoviscosity genes like magA, rmpA, aerobactin but the exact mechanism by which this spreading take place is unknown. ${ }^{13}$ Shon et al. observed that hvKP was resistant to complement- and neutrophil-mediated bactericidal activity in a rat subcutaneous abscess model and produces more biofilm than others strains. ${ }^{14}$ This capacity increases intestinal colonization. In this case, this patient developed KLAS even if he had been colonized in an endemic area several years ago. Colonization usually happens when there is a disruption of the natural barriers (ascension into the bladder, aspiration into the respiratory tract, gastrointestinal colonizers) but KLAS has been reported in people, like this patient, without evidence of altered mucosal barriers. ${ }^{4}$

Even though the majority of patients with hvKP infection are healthy, there is a significant mortality rate between 3 and $42 \%$ in part following necrotizing fasciitis and severe communityacquired pneumonia. ${ }^{1,4,14}$ Moreover, survivors can suffer appalling consequences such as blindness and neurologic sequelae. ${ }^{14}$

Even if most hvKP infections are treatable with common antibiotics, long-term follow-up is necessary because of the high risk of relapsing. Several cases of resistant strains with extended spectrum B-lactamases and carbapenemases have already been reported. ${ }^{4,5,14,15}$ All these characteristics could make hvKP one of the next "superbugs."

\section{Conclusion}

To our knowledge, this is the first report of a complete metastatic syndrome by HvKp in Canada, following the first published case of pyogenic liver abscess caused by hvKP in Manitoba in 2007. ${ }^{10}$ Compelling questions about hvKP remain unanswered. Its natural course is barely understood. Diagnostic features such as a positive 'string test' and capsular serotypes like K1 or K2 are not always distinctive of hvKP. ${ }^{16}$ Its capacity for metastatic spread, an unusual trait for an enteric gram-negative, is a worrying characteristic of this strain of Klebsiella that is no longer confined to Asia.

\section{References}

1. Siu L Kristopher, Yeh Kuo-Ming, Lin Jung-Chung, et al. Klebsiella pneumoniae liver abscess: a new invasive syndrome. Lancet Infect Dis 2012;12(11):881-87.

2. Nadasy KA, Domiati-Saad R, Tribble MA. Invasive Klebsiella pneumoniae syndrome in North America. Clin Infect Dis 2007;45:e25-e28. 
3. Coutinho RL, Visconde MF, Descio FJ, et al. Community-acquired invasive liver abscess syndrome caused by a K1 serotype Klebsiella pneumoniae isolate in Brazil: a case report of hypervirulent ST23. Mem Inst Oswaldo Cruz 2014;109(7):970-71.

4. Shon A, Bajwa R, Russo T. Hypervirulent (hypermucoviscous) Klebsiella pneumoniae. Virulence 2013;4(2):107-18.

5. Jung-Chung Lin, Feng-Yee Chang, Chang-Phone Fung, et al. High prevalence of phagocytic-resistant capsular serotypes of Klebsiella pneumoniae in liver abscess, Microbes Infec 2004;6(13):1191-98.

6. Kuo-Ming Yeh, Jung-Chung Lin, Fon-Yi Yin, et al. Revisiting the importance of virulence determinant magA and its surrounding genes in Klebsiella pneumoniae causing pyogenic liver abscesses: exact role in serotype K1 capsule formation. J Infect Dis 2010;201(8):1259-67.

7. Soon WC, Pouncey A, Ashley E, Bowen EF. Klebsiella pneumoniae infection: a virulent cause of visual loss. Case Rep Ophthalmo 2014;5(3):468-73.

8. Carrillo Esper R, Soto Hernandez JL, Peña Pérez CA, et al. Sindrome de absceso hepatico secundario con involucro pulmonar. Gac Méd Méx 2013;149(1):102-107.

9. Gundestrup S, Struve C, Stahlhut SG, Hansen DS, et al. First case of liver abscess in scandinavia due to the international hypervirulent klebsiella pneumoniae clone ST23. Open Microbiol J 2014;8:22-4.

10. Yoav K, Karlowsky JA, Walus T, et al. Pyogenic liver abscess caused by hypermucoviscous Klebsiella pneumoniae. Scand J Infect Dis 2007;39(9):828-30.
11. Anstey JR, Fazio TN, Gordon DL, et al. Community-acquired Klebsiella pneumoniae liver abscesses - an "emerging disease" in Australia. Med J Aust 2010;193(9):543-5.

12. Cheng D, Liu Y, Yen M, Liu C, et al. Septic metastatic lesions of pyogenic liver abscess: their association with klebsiella pneumoniae bacteremia in diabetic patients. Arch Intern Med 1991;151(8):1557-59.

13. Russo TA, Olson R, MacDonald U, et al. Aerobactin mediates virulence and accounts for increased siderophore production under iron-limiting conditions by hypervirulent (hypermucoviscous) klebsiella pneumoniae. Infect Immun 2014;82(6):2356-67.

14. Shon AS, Russo TA. Hypervirulent klebsiella pneumoniae: the next superbug? Future Microbiol 2012;7(6):669-71.

15. Rafiq Z, Sam N, Vaidyanathan R. Whole genome sequence of Klebsiella pneumoniae U25, a hypermucoviscous, multidrug resistant, biofilm producing isolate from India. Mem Inst Oswaldo Cruz 2016;111(2):144-46.

16. Yu WL, Ko WC, Cheng KC, Lee CC, et al. Comparison of prevalence of virulence factors for Klebsiella pneumoniae liver abscesses between isolates with capsular K1/K2 and non-K1/K2 serotypes. Diagn Microbiol Infect Dis 2008;62(1):1-6.

17. Ting-ting Q, Jian-cang Z, Yun-song Y, et al. Clinical and microbiological characteristics of Klebsiella pneumoniae liver abscess in East China. BMC Infect Dis 2015;15(1):161.

18. Setsu T, Tsuchiya A, Yamagiwa S, et al . Invasive liver abscess syndrome caused by Klebsiella pneumoniae. Intern Med 2017;56(22):3121-22. 